\title{
Soziologie, soziale Arbeit und Frauenbewegung - eine Art Familiengeschichte
}

Heute hat innerhalb der Soziologie die empirische Sozialforschung einen ungeheuren Aufwind zu verzeichnen. Als »echte Soziologie " gilt fast nur noch die Arbeit »im Feld «. Glaubt man den soziologischen Wörterbüchern, dann wurde die empirische Sozialforschung in der Bundesrepublik Deutschland aber erst nach dem Zweiten Weltkrieg aus den USA importiert. In den 20er und 30er Jahren habe sie sich insbesondere in Chicago herausgebildet und sei von dort nach Europa gekommen. Entsprechendes wird noch in neueren Nachschlagewerken behauptet - soweit sie die Geschichte der Disziplin überhaupt berücksichtigen, denn die Nachkriegssoziologie ist eine im wesentlichen ahistorische Soziologie. Der »Paradigmenwechsel« nach 1968 hat diese Tendenz eher verstärkt denn geschwächt.

Allerdings war die ältere deutsche Universitäts-Soziologie vornehmlich eine theoretische Soziologie. Es entspricht der Disziplinhistorie, wenn die einschlägigen Darstellungen ihrer Geschichte die Soziologie vor allem als Geschichte soziologischer Ideen präsentieren, die im wesentlichen aus der großen "sozialen Frage « des 19. Jahrhunderts geboren, direkt in die theoretischen Debatten mündete.

Aber es läßt sich auch eine andere Genealogie der Soziologie rekonstruieren und zwar die Entstehung der Soziologie aus einer - sagen wir »wallraffartigen « Sozialreportage. Diese Soziologie vereinte in einem Erfahrungsansatz Empirie und Theorie, eine »Stadtsoziologie«, die bis in die »Kultursoziologie« reicht und in der ethnographischen Beschreibung aufgrund von teilnehmender Beobachtung vor allem offene Augen für alle mikrosozialen Verhältnisse hat. Diese Form von Soziologie entstand nicht voraussetzungslos.

Entscheidend war ihr Entstehen im Anschluß an engagierte praktische Versuche zur Sozialreform, die dem engagierten Journalismus vorausging. In den sozial sehr interessierten 1890er Jahren standen im Mittelpunkt des allgemeinen Interesses vor allem Chicago, London und Berlin. Hier brachten Praktikerinnen aus dem Umkreis der ersten Frauenbewegungen den Forschern soziale Probleme konkret nahe. Feministinnen und Sozialreformer erfanden die "soziale Arbeit«, in der sie sich den einzelnen Elenden ihrer Zeit mit "pädagogischen « Absichten zuwandten. Sie beschrieben ihre Erfahrungen, regten Enquêten an und richteten Schulungsprogramme für jüngere Helferinnen ein. Als Lehrer für die von ihnen 
neu gegründeten Hochschulen für Sozialarbeit engagierten sie Vertreter der entstehenden Soziologie.

Diese Gründervätergeneration der Soziologie ging gerne zu den Frauenrechtlerinnen, denn deren Berichte stammten unmittelbar aus der Erfahrung der sozialen Praxis in den Armenvierteln selbst. Um diesen bis heute seitens der Fachhistoriographie verdrängten Entstehungszusammenhang moderner Sozialforschung soll es in diesem Text gehen.

\section{Anfänge in Chicago}

Zur Zeit der Jahrhundertwende arbeitete in Chicago Robert E. Park (1864-1944), ein Altersgenosse von Max Weber. Robert Park war lange als Journalist tätig, später für den ersten afroamerikanischen Abgeordneten. Schließlich landete er an der Universität. Hier erklärte er das »nosing around « zu seiner Methode. Robert Park geht es um die genaue Beschreibung der den Etablierten fremden Lebenswelten der Armen, der wertfreien Schildenung der Unterwelten, vom Lebenslauf eines Diebes bis hin zu dem eines obdachlosen Tramps. So entwickelte er eine neue Stadtsoziologie aus der Methode der teilnehmenden Beobachtung.

Vorläufer dieser Soziologie waren sogenannte »Enthüllungsreportagen « von Reportern wie etwa Elizabeth Coranche (geb. 1867; Psydoneum: Annie Laurie). Sie bediente sich in ihren Studien in den 1890er Jahren bereits einer Methode der verdeckten teilnehmenden Beobachtung. Sie gab geistige Verwirrung vor und ließ sich in ein berüchtigtes Irrenhaus einweisen, um anschließend darüber berichten zu können. Sie ließ sich verhaften, um über ein Frauengefängnis berichten zu können oder durchwanderte die Stadt verkleidet als Angehörige der Heilsarmee (Rolf Lindner 1990, 34).

Eine Form dieser Erhebungen war das »muckraking «, das Abfassen sozialkritischer Artikel, die auf »harten « Fakten beruhten. Die Anfänge dieser Reportagen kamen von Josiah Flynt Willard, der Artikelfolgen zu »The true Stories from the Underworld « schrieb. Ihm ging es darum, Schiebungen zwischen Ganoven, Politikern, Polizei und Richtern aufzudecken. Er war der Neffe einer der Pionierinnen sozialer Reformen in den USA und bei ihr aufgewachsen. Er veröffentlichte wahrscheinlich mit Rücksicht auf seine Ziehmutter unter seinem »middelname « Flynt (Rolf Lindner 1990, 42f.).

Sowohl Flynt als auch Park war es wichtig zu betonen, daß es ihnen um wertfreie Erhebung ging, nicht jedoch um unmittelbare Sozialreform. Die direkte Praxisorientiertheit der Anfänge der Chicagoer Soziologie der Jahrhundertwende lehnten sie ab. Sie wandten sich damit gegen die ersten Chicagoer Sozialuntersuchungen der $1890 \mathrm{er}$ Jahre, die in engem Zusammenhang mit der praktischen Sozialreform gestanden hatten und vielfach von Frauen unternommen worden waren. 


\section{Frances Willard und die neue Sozialarbeit}

1874 war nach dem Bürgerkrieg und einem »Frauenkreuzzug « gegen den übermäBigen Alkoholkonsum im Mittleren Westen der USA eine Woman's Christian Temperance Union, eine weibliche Mäßigkeits-Organisation, entstanden. Diese WCTU wurde binnen kurzem zu einem der größten Frauenvereine, die es je gab (Ruth Bordin 1981, 94). Hinter dieser ersten Antialkoholbewegung der Frauen verbarg sich eine Art erste »Frauenhausbewegung " und mehr als das. Damals vermochten die Frauen noch nicht offen von ihrer oder der Schande anderer Frauen zu sprechen. Die Säufer ließen die Familie hungern, verprügelten Ehefrauen samt Kindern und hintertrieben die Familienplanung (Elisabeth MeyerRenschhausen 1989, $171 \mathrm{ff}$.).

Diese Frauenorganisation, die von Anfang an Männer ausschloß, wurde binnen kurzem zu einem der radikalsten Frauenvereine der Vereinigten Staaten, die selbstverständlich auch African Americans aufnahm und sich für Minderheiten einsetzte. Die WCTU führte Frauen aller sozialen Schichten aus der kirchlichen Armenpflege in eine »weltliche « soziale Arbeit. In den 1880er Jahren wurde die WCTU zur Anregerin und Schöpferin wichtiger weltlicher sozialer Hilfseinrichtungen, wie sie der Einwandererstaat USA bislang kaum gekannt hatte. Diese soziale Arbeit ließ in den USA in den 1880er und 1890er Jahre bald eine größere Frauenbewegung entstehen, die nach 1900 in eine Wahlrechtskampagne mündete.

Die herausragende Persönlichkeit innerhalb dieser Organisation war Josiah Flynts Tante, Frances Willard (1839-1898), eine ehemalige College-Professorin, die ihr College - nachdem ihr eine anstehende Beförderung versagt worden war - verließ, um sich ab 1873 ganz der Arbeit innerhalb der Union zu widmen. Unter ihrer Ägide wurde aus der WCTU eine Frauenorganisation, die auf fast allen Gebieten sozialer Reform aktiv war. Frances Willard prangerte in ihren Reden Doppelmoral, Pressemacht und Lynchjustiz an, forderte den Acht-Stundentag, das Wahlrecht für alle, eine Ernährungsreform, Solidarität mit der Arbeiterbewegung und soziale Reformen sowie Hilfen für das hungernde Armenien und einen internationalen Schiedsgerichtshof zur Eindämmung aller Kriege. Sie wurde so zu der Identifikationsfigur der weiblichen Jugend Amerikas jener Tage (Ruth Bordin $1981,151,157)$.

Bereits 1880 war die Union die größte Frauenorganisation, die jemals in den USA existiert hat. In fast jeder Stadt gab es Zweigvereine, die nach der Parole der Vorsitzenden Frances Willard »do everything « verschiedenste soziale Einrichtungen ins Leben riefen. Dadurch kamen die Frauen mit den Kommunalbehörden und den Stadtparlamenten in Kontakt und wurden zu einem wichtigen Motor für soziale Reformen.

In der zweitgrößten Stadt der USA, in Chicago, wo soziales Elend, Gangsterbanden, soziale Unruhen wie die vom Haymarket und Streiks grassierten, hatte die WCTU besonders viele engagierte Mitglieder. 1889 hatte die WCTU dort bereits zwei Kindertagesstätten, zwei Sonntagsschulen, ein Billig-Restaurant, eine Industrie-Schule, eine Herberge für wohnungslose Frauen, ein Obdachlosenasyl für Männer und eine kostenlose Ambulanz für Unbemittelte eingerichtet und außerdem Heime für »gefallene « Mädchen unter weiblicher Leitung 
mit ausschlieBlich weiblichen Mitarbeiterinnen angeregt (Ruth Bordin 1981, XIII ff., 98).

So wundert uns nicht, daß die erste weibliche Fabrikinspektorin in den USA, Florence Kelley, 1893 hier in Illinois angestellt wurde (Florence Kelley 1897, 129f.). Ihren Berichten entnehmen wir, warum Chicago so exponiert war: Chicago war damals eine der "neuesten « und am rapidesten wachsenden Städte, Zentrum einer der Haupt-Einwanderer-Regionen der USA. Ende des 19. Jahrhunderts waren mehr als die Hälfte der Einwohner der Stadt Immigranten, die oft noch kein Englisch konnten. Die verelendeten Gruppen bestanden fast ausschließlich aus gerade neu hinzugekommenen Einwanderern. Die Stadt bestand aus einem Konglomerat von ethnischen Minderheiten.

Besonders große Einwandererschübe, wie der der galizischen und russischen Juden, die Europa auf der Flucht vor Pogromen verlassen hatten, machten neue Ausbeutungs-Systeme wie das Sweating-System möglich, das der älteren »Küchenschneiderei« das Wasser abgrub (Florence Kelley 1898). Als in den 1890er Jahren die wachsende Arbeitslosigkeit zu Mißmutäußerungen gegenüber Neueinwanderern führte, beriet die Regierung in Washington ein Einwanderergesetz. Aber in ihrer Untersuchung zu der jüngsten Einwanderergruppe in Chicago, den Italienern, stellte Florence Kelley fest, daß sie nahezu ausschließlich die äußerst schlecht bezahlten Jobs wie die der Straßenkehrer oder Tunnelgräber hatten, die die schon länger in der Stadt wohnenden Gruppen für sich mittlerweilen strikt ablehnten (Florence Kelley 1899).

Wenn Park in seiner Darstellung der in seinen Augen relevanten empirischen Soziologie mit dem Beispiel Little Italy in North Chicago beginnt, ist das wohl kein Zufall. Er wird Florence Kelleys Studie vor Augen gehabt haben. In den USA lernte die soziologische Feldforschung, nebenbei bemerkt, schnell von der Anthropologie, insofern die Neueinwanderer lauter Minderheitenviertel bildeten, die in ihren Eigenarten - zumal aller Komfort ja noch fehlte - Dörfern von "Stammesgesellschaften « durchaus vergleichbar waren. Insofern ist der Beginn der empirischen Sozialforschung "naturwüchsig " mit der Frage nach Minderheiten verknüpft.

\section{Jane Addams und das Settlementhouse}

Die Einrichtung der weiblichen Fabrikinspektion in Illinois war das Ergebnis einer Kampagne gegen das Sweating-System als extremem Ausbeutungssystem. In Gang gesetzt worden war sie vom Hull-House, einer bald weithin bekannten sozialen Einrichtung in Chicago. 1889 waren Jane Addams (1860-1935), ihre Freundin Ellen Starr und ihre Haushälterin Mary Keiser in das Gebäude einer alten Dame namens Hull-House gezogen, um dieses als Nachbarschaftsheim einzurichten (C. Wolfgang Müller 1982, 86). Damit war der Grundstein für eine völlig neue "soziale Arbeit « gelegt worden, die den Schwerpunkt von der bloßen Unterstützung weg auf eine neue Art »sozialer Pädagogik « verlegte.

Dieses Settlementhouse strebte nämlich keine karitative Arbeit an, sondern stand der gesamten Nachbarschaft offen: zur Erholung, für Bildungs-Veranstal- 
tungen oder als Räumlichkeit, in der beispielsweise Gewerkschaftsvereine gegründet werden konnten. So ging Mary Abderson, eine Schuharbeiterin, ins HullHouse nicht nur zu ihren Gewerkschaftsversammlungen, auf denen gelegentlich auch Jane Addams sprach, sondern mit Kolleginnen auch zu Jane Addams Sonntagsnachmittags-Tees, wo sie Sozialreformer/innen aus fast dem gesamten In- und Ausland kennenlernte. Für die Schuharbeiterin wurde - wie sie es selbst formulierte - das Hull-House das Tor zur Welt. Mary Abderson wurde später Direktorin der Frauenabteilung des amerikanischen Arbeitsministeriums (Eleanor Flexner 1978, 257).

Jane Addams nannte dieses Haus ein »social settlement « und tatsächlich taucht in diesen Jahren der Begriff »social work «, »soziale Arbeit «, erstmals auf, um bald weltweit aufgegriffen zu werden. Das Settlementhouse zog junge Frauen und Männer von überall an, die nicht selten später wichtige Positionen im Bereich der Sozialpolitik bekleideten. Jane Addams meinte einmal, daß sie eine Generation junger Amerikaner von Philanthropen zu Reformern gemacht habe. Eine Mitarbeiterin des Hull-House, Sophnisba Breckinridge, wurde Dekanin der "Schule für Staatsbürgerkunde und Philanthrophie«, der späteren Schule für Sozialarbeit an der Universität von Chicago. Um 1900 gab es bereits 100 solcher Gründungen in den USA (Wolf Rainer Wendt 1985, 150).

Vom Hull House ging die Anregung zu sozialen Reformen auf allen Ebenen aus, wichtig waren empirische Untersuchungen. Ein 1890 gegründeter »Working People's Social Science Club « und das Settlement erarbeiteten erstmals Studien mit genauen Daten über die Not unter den Arbeitern und Armen in Chicago. Diese Untersuchungen (darunter mehrere von Florence Kelley) wurden ab 1895 in den "Hull-House Maps and Papers" veröffentlicht. Sie gelten als eine Wurzel empirischer Sozialforschung.

Die Chicagoer Frauengruppe regte außerdem eine vom Kongreß finanzierte Erhebung über die Arbeits- und Lebensbedingungen von Arbeiterinnen in den USA an. Die Studie - durchgeführt von 1911 bis 1915 - wurde zu einem 19bändigen Bericht, der die Grundlage der neuen Arbeiterinnenschutzgesetzgebung war (Eleanor Flexner 1978, 259). »Die ... objektive, wissenschaftliche Erforschung sozialer Bedingungen war erforderlich, um das allgemeine Interesse an Reformen zu fördern« (Walter Goldschmidt 1968, 226).

Im Jahr 1894 gründete die Universität von Chicago ein eigenes "Settlementhouse « im Slum »Hinter den Höfen«. Eine Freundin Frances Willards, Mary McDowell, die seit 1871 in der sozialen Arbeit Chicagos aktiv war und zu den ersten Mitarbeiterinnen des Hull-House gehört hatte, wurde die Leiterin (Eleanor Flexner 1978, 257). Dieses Settlementhouse sollte dem ersten soziologischen Institut als »Fenster « auf die soziale Realität dienen. Die Universitäts-Soziologie entstand also mitten in der Hochburg der amerikanischen sozialen Arbeit und Pädagogik. Es ist nicht erstaunlich, daß die Chicagoer Soziologie den Kommunalpolitikern empfahl, die Stadt in neighbourhood communities zu planen. Dieses Modell war in gewisser Weise der Realität der Einwandererstädte selbst, die in ethnic quarters aufgeteilt war, entnommen. 


\section{Die Barnetts und die Webbs in London}

Die Gründerin des Hull-House, Jane Addams, wurde durch eine ältere damals bereits weltweit bekannte Gründung in London, die Toynbee Hall, zu ihrer Initiative anregt. Seit Mitte der 1860 er Jahre hatten sich Pfarrer gezielt ins arme East London einsetzen lassen, um von dort aus soziale Reformen vorzuschlagen. 1884 hatte das Pfarrerehepaar Henriette und Samuel Barnett zu diesem Zweck eine Pfarrei in Whitechapel übernommen. Dort gründeten sie die Toynbee Hall als Settlementhouse. Hier wohnten an sozialen Reformen interessierte Studenten direkt unter den Armen und halfen dem Pfarrerehepaar, verschiedene Clubs einzurichten, in denen den Armen Bildung und Ausbildung vermittelt wurde. Dazu gehörte der wöchentlich einmal stattfindende offene Abend im Hause der Barnetts, wo die Obdachlosen und Bettler zu einem Abendessen mit Vortrag, Rezitation und Unterhaltung eingeladen wurden. Meistens zogen bis zu 20 junge Leute für etwa ein Jahr als »residents « zusammen, um sich mit diesem »sozialen Jahr « für Berufe in der Verwaltung vorzubereiten und in der Öffentlichkeit ein Bewußtsein für soziale Reformen herzustellen. Bereits 1900 gab es zwanzig solcher SettlementHäuser in London, von denen immer mehr von Frauen bezogen und bewohnt wurden (Wolf Rainer Wendt 1985, 146). Parallel zu dieser praktischen Kritik an der älteren Philanthropie führte die neue Sozialforschung umfassende Sozialerhebungen mittels Befragungen von Sachverständigen, Betroffenen und ergänzender teilnehmender Beobachtung ein.

1899 berichtete der Züricher Nationalökonom Heinrich Herkner begeistert von der ersten Generation von Frauen im Wissenschaftsbetrieb. Besonders bekannt wurde die Nationalökonomin Beatrice Webb, geborene Potter (1858-1943). Sie stammte wie ihr Mann Sidney James Webb (1859-1947) aus der praktischen Sozialreformarbeit, wo sie in engem Kontakt mit den Barnetts gestanden hatte. "Im Vereine mit ihrem [angeheirateten] Vetter Charles Booth [1840-1916] faßte sie den Plan, ... die thatsächlichen Zustände der Arbeiterbevölkerung, zunächst derjenigen im Londoner Ostende, einmal gründlich zu untersuchen« (Heinrich Herkner 1899). Sie schlug Booth vor, durch Befragung von Inspektoren der Schulverwaltung, die nach fehlenden Kindern fahndeten, sich Einblicke in die Lebensverhältnisse von Arbeitern zu verschaffen und mietete sich selbst als Näherin bei Arbeitern ein, um deren Leben aus nächster Nähe mitzubekommen. Sie trug zu Booths Untersuchungen mit einer Studie über die jüdischen Einwandererfamilien aus Polen und Rußland bei. Zusammen mit Sidney Webb entwickelte sie eine Soziologie der genauen Beschreibung sozialer Verhältnisse. Beide arbeiteten in der 1884 gegründeten Fabian Society, die für entschiedene Gesellschaftsreform eintrat und Beraterin der Independent Labour Party wurde. 1895 gründeten die Webbs die London School of Economics (Wolf Lepenies 1985). Die zweite Professur für Soziologie wurde folgerichtig 1907 in London eingerichtet. 


\section{Frauenbewegung und Kathedersozialisten}

Auf großen internationalen Ausstellungen und zahlreichen Kongressen gab die Frauenbewegung einschließlich der weiblichen Temperenzbewegung ihre Erfahrungen weiter. Anläßlich der Weltausstellung 1893 in Chicago hatten die dortigen Aktivistinnen einen ersten Internationalen Frauenkongre $\beta$ einberufen. Auf diesem Weltkongre $B$ der Frauen wurde die Gründung von nationalen Frauenverbänden als Dachorganisationen aller sozial arbeitenden Frauengruppen in den jeweiligen Staaten angeregt. Die Gründung eines entsprechenden Bundes Deutscher Frauenvereine erfolgte in Berlin bereits 1894. Auch im deutschen Kaiserreich hatten riesige Migrationsbewegungen vor allem von Ost nach West, nach Berlin, ins Ruhrgebiet zu Slums am Rande der Großstädte geführt. Seither hatte die seit 1865 organisierte Frauenbewegung sich auch stark im Bereich der praktischen Sozialreform engagiert.

Die entstehende Soziologie griff die Anregungen der Sozialreformerinnen auf. Georg Simmel, dessen Frau und dessen Freundin dem Umfeld der Frauenbewegung zuzurechnen sind, veröffentlichte 1906 ein Essay über den Armen. Der Arme als soziologischer Begriff entstehe überhaupt erst durch die Art der Zuwendung, meinte er und kritisierte damit das alte System städtischer und karitativer »Armenpflege " (Georg Simmel 1908, 490; Robert Jütte 1991). Er griff damit die Kritik der Frauenvereine und der Settlementhousebewegung an der alten Armenversorgung auf. Die Barnetts hatten sich 1886 von der noch gar nicht alten philanthropischen Charity Organisation Society (C.O.S.) getrennt. Ihnen ging es um Hilfe zur Selbsthilfe, das Ausspionieren der Hilfeempfänger, wozu die private Wohltätigkeit trotz anderer Absichten staatlicherseits gezwungen worden war, lehnten sie ab (Beatrice Webb 1988, 250). Sie legten so den Grundstein zu einer Gemeinwesenarbeit, die das alte System der entmündigenden Armenfürsorge strikt ablehnte und durch eine beratende Fürsorge von ausgebildeten »sozialen Arbeiterinnen « zu ersetzen suchte.

Der traditionelle Arme, so Simmel, wird wie der klassische Fremde behandelt: er hat keinen »angeborenen« Anspruch auf Hilfe, wird im Zweifelsfall sogar davon gejagt. Wenn er Hilfe bekommt, geht es nicht um ihn als Person, sondern um »übergeordnete Interessen« (Georg Simmel 1906). Die zeitgenössischen Reformer und Reformerinnen empfanden diese Auffassung vom Armen in einer Gesellschaft, die auf Zuwanderung vom Umland in die Städte, aus den armen Regionen Osteuropas in die industriellen Zentren des Westens angewiesen war, einer Industriegesellschaft, die immer weitere Verarmung von Bauern und neue Wellen von Landflucht erzeugte, als überholt. Es ging ihnen darum, die Armen, und das hieß fast immer die Neueinwanderer und Flüchtlinge, aus ihrem marginalisierten Status zu befreien.

1863 und 1865 waren reichsweite Arbeitervereine gegründet worden, die "Soziale Frage « war damit sozusagen »institutionalisiert«. 1872 wurde in Halle ein ebenfalls überregionaler Verein für Sozialpolitik gegründet. Er richtete sich gegen den »Manchesterliberalismus « des 1858 entstandenen Kongresses der Volkswirte und plädierte für soziale Reformen. Von den Manchesterliberalen wurden die Hochschullehrer dieser Richtung daraufhin mit dem Spottnamen »Kathedersozia- 
listen « abgekanzelt. Die Gründungsmitglieder des Vereins für Sozialpolitik hielten Reformen für notwendig, da andernfalls die drohende Revolution der Arbeiterklasse nicht zu verhindern sei. Zur allgemeinen Wohlfahrt des Staates gehörten ihrer Meinung nach erträgliche Lebensumstände für alle, für die sich der Staat einzusetzen habe (Else Conrad 1911; Wilhelm Lexis 1910).

Der Verein befaßte sich in den ersten zehn Jahren seines Bestehens insbesondere mit der »sozialen Frage « und der Arbeiterbewegung. Nach 1890, als nach Aufhebung der Bismarck'schen Sozialistengesetze (1878-1890) fast niemand mehr eine staatliche Sozialpolitik ablehnte, trat der Verein wieder offen für soziale Reformen ein. Jetzt trat eine neue Forschergeneration auf den Plan wie unter anderen Werner Sombart und Werner Tönnies. Von ihnen verstanden sich viele insofern zeitweilig als »Marxisten «, als sie sich im Gegensatz zur älteren Generation mit Marx gezielt auseinandersetzten, mit dem Ergebnis, daß sie in ihren Theorien in immer größere Distanz zu Marx gerieten. Der dem Verein 1888 beigetretene Max Weber galt bald als Antipode zu Marx. Diese »Jüngeren « oder "Linken « hielten den Glauben an einen neutralen Staat seitens der älteren Vereinmitglieder um Gustav Schmoller, Adolph Wagner oder Lujo Brentano für falsch. Sie waren statt dessen an einer direkten Auseinandersetzung mit der Arbeiterbewegung interessiert.

1892 gründete Jeanette Schwerin, geb. Abarbanell (1852-1899) nach New Yorker Vorbild in Berlin eine Gesellschaft für ethische Kultur (Wolf Rainer Wendt 1985, 144). Im Rahmen dieser Gesellschaft schuf sie 1893 eine zentrale Kartei sowohl für Hilfesuchende als auch potentielle Helfer/innen. Diese Auskunftsstelle des Vereins für ethische Kultur gab bald ein umfassendes Handbuch aller freien Wohlfahrtseinrichtungen Berlins heraus. Daraus entstand später die Zentralstelle der freien Wohlfahrtsverbände (Elisabeth Meyer-Renschhausen 1989, 135 ff.).

Im gleichen Jahr 1893 gründeten Frauen des Berliner Vereins Frauenwohl die Mädchen- und Frauengruppen für soziale Hilfsarbeit, die schnell zum Zentrum der weiblichen sozialen Hilfsarbeit im Reich wurden. Es ging darum, die Helferinnen für ihre Aufgaben in der praktischen Arbeit so zu schulen, daß sie nicht unwissend und voller Vorurteile in die Armenviertel gingen. Aus den Reihen der »Kathedersozialisten« wurden Lehrer für die entsprechenden Kurse gewonnen. Redner wurden gleich im ersten Jahr außer den Sozialdemokraten Heinrich von Braun (dem zweiten Mann Lily Brauns) und Leo Katzenstein auch Max Weber und Emil Münsterberg (Alice Salomon 1983, 31 ff.). Aus diesen Kursen der Mädchen- und Frauengruppen entstand unter der Leitung Alice Salomons (1872 -1948) 1908 die erste Soziale Frauenschule, also die erste der späteren »Hochschulen für Sozialarbeit und Sozialpädagogik «.

Im Umkreis der Mädchengruppen entstanden jede Menge neuer »Projekte«, wie wir es heute nennen würden. Während die Forderungen der Arbeiterbewegung am Lohnarbeiterstatus ansetzten und zunächst zu verbesserten Arbeitsschutzgesetzen führten und auch die staatliche Sozialpolitik mit der Bismarckschen Sozialgesetzgebung nur die Lohnarbeiter erfaßte, setzten die Frauen dort an, wo die alte private Hilfe für »verschämte Arme « aufgehört hatte: bei der privaten Hilfe außerhalb des staatlichen Armenpflegesystems. Die Adressaten der Frauenvereine waren insbesondere überlastete, unterbezahlte Familienmütter und ihre unbeaufsich- 
tigten oder mitarbeitenden Kinder. Waren doch Bedürftige, die keine Lohnarbeiter waren, und ganz besonders familienlose Bedürftige in viel größerer Gefahr, Opfer wtotaler Institutionen « zu werden, als jeder noch so kleine Familienverband. Nach dem Ersten Weltkrieg wurde das alte Armenpflegesystem auf Initiative von Frauen aller Parteien durch eine völlig neue Wohlfahrtsgesetzgebung (nebst einem Reichsjugendwohlfahrtsgesetz) ersetzt, in deren Zentrum die »Familienfürsorge " stand (Marie Baum 1927). Da der Staat aber keine angemessenen Mittel bereitstellte, vielmehr im sozialen Bereich sparte, wo es nur ging und auch da, wo es nicht ging, geriet die neue »Fürsorge« früher oder später in ähnlichen Verruf wie die alte Armenpflege.

\section{Gebremst von der Bürokratie}

Max Weber bekam diese Kämpfe der Frauenbewegung hautnah mit: Seine Mutter, Helene Weber, geb. Fallenstein (1844-1919), war sozial aktiv. Sie kochte für schwangere Arbeiterinnen am eigenen Herd und sammelte Kleider, die sie an bedürftige Familien verteilte. Ihre Initiative führte zu einem Hauspflegeverein, einem Jugendheim und schließlich einer Wohlfahrtszentrale für Berlin-Charlottenburg (Ingrid Gilcher-Holtey 1988, 143). Sie setzte sich - damals keine Selbstverständlichkeit - für uneheliche Kinder ein und wurde 1904 zur ersten Stadträtin Berlins (Martin Green 1976, 129). Max Weber, der 1886 nach seinem Studium wieder ins Elternhaus nach Charlottenburg zurückkam, mußte miterleben, wie diese Aktivitäten seiner Mutter die Spannungen zwischen den Eltern wachsen ließen. Er ergriff Partei für die Mutter. 1892 nahm Helene Weber Marianne Schnitger (1870-1954) ${ }^{1}$ aus der westfälischen Verwandtschaft der Webers, Max Webers spätere Frau, bei sich auf und unterstützte die junge Frau bei ihrem Vorhaben zu studieren. Während ihres Studiums in Freiburg lernte Marianne Weber Else Richthofen, später verheiratete Jaffé, kennen. Nach ihrem Umzug nach Heidelberg 1897 gründete Marianne Weber dort einen Verein zur Ausbreitung der modernen Frauenideale, in dessen Versammlungen es oft hoch her ging und Max Weber beredt für die Ziele der Frauenbewegung eintrat (Marianne Weber 1926, 241 f.). Durch Mariannes Aktivitäten kamen die Webers in engen Kontakt mit den Wortführerinnen und Praktikerinnen der damaligen Frauenbewegung. Marianne Weber, der wir die Herausgabe eines Gutteils von Max Webers Schriften verdanken, war befreundet mit Marie Baum, Gertrud Simmel, Camilla Jellinek, Gertrud Bäumer und Helene Lange. Max Weber war kurzzeitig sogar Mitglied des Bundes für Mutterschutz, Marianne Weber nach dem Krieg 1918 bis 1923 Vorsitzende des Bundes Deutscher Frauenvereine (Marianne Weber 1926, 376 ff.).

Die Frauenbewegung war durch wenn auch konfliktreiche Zusammenarbeit mit dem 1880 gegründeten Deutschen Verein für Armenpflege und Wohltätigkeit nach jahrelanger Vorarbeit um 1900 an dem Punkt, dem Staat oder genauer den Kommunen erste Zugeständnisse abringen zu können. Die Ergebnisse waren ambivalent. Wohl gestand der Staat den Frauen kleine, meist unbezahlte Ämter zu, erstickte auf der anderen Seite aber fast alle Bemühungen der Frauen in einem Wust von bürokratischen Vorschriften wie auch durch Nichtbereitschaft, für ge- 
ringste materielle Hilfestellungen Sorge zu tragen. Statt dessen wurde den Praktikerinnen verboten, aus ihrer Praxis zu berichten. ${ }^{2}$

Else Richthofen, die enge Freundin Mariannes und Max Webers, übernahm nach ihrem Studium 1899 als erste Frau im Kaiserreich die Aufgabe einer weiblichen Fabrikinspektorin. Die Anstellung von Fabrikinspektorinnen hatte die deutsche Frauenbewegung nach englischem und amerikanischem Vorbild seit Jahren gefordert. ${ }^{3}$ Die Frauen hofften, so unhaltbare Zustände aufdecken und damit zu Reformen anregen zu können. Else Richthofen hielt dieses schwierige Amt zwischen »Baum und Borke « kaum aus und floh via Eheschließung mit dem Heidelberger Dozenten Edgar Jaffé zurück zu den Büchern. Nach der Trennung von ihrem Mann lebte Else Jaffé ab 1910 jahrzehntelang mit Alfred Weber zusammen und half ihm bei den Exzerpten zu seiner »Kultursoziologie«, die er ihr widmete. Ihre Nachfolgerin im Amt wurde die ehemalige Züricher Studentin, Assistentin, Berliner Chemielaborantin und Freundin Ricarda Huchs, Dr. Marie Baum (1874-1964).

Auch Marie Baum kam aus einer Familie, in der die Mutter "sozial« arbeitete. Marie Baum stürzte sich mit Feuereifer in die Arbeit. 1906 erschien ihre Untersuchung »Drei Klassen von Lohnarbeiterinnen in Industrie und Handel der Stadt Karlsruhe«. Diese Untersuchung ist noch heute interessant, weil die ländlichen »Umlandgemeinden « der Stadt eben erst in den Industrialisienungs- und Verstädterungsproze $\beta$ einbezogen wurden. Marie Baum stellte fest, da $B$ die Fabrikarbeit von den jungen Mädchen aus den ländlichen Gemeinden zwar mit der Heirat zugunsten der Nebenerwerbslandwirtschaft aufgegeben wurde, aber dazu führte, $\mathrm{da} \beta$ die jungen Frauen kaum noch Haushaltswissen erwerben konnten, was sich in einer relativ hohen Kindersterblichkeit bemerkbar machte. Der Wunsch der Landmädchen, vor der Eheschließung in »Dienste « gehen zu können, um so das „Wirtschaften« erlernen zu können, erfüllte sich meistens nicht mehr (Marie Baum $1906,182 \mathrm{ff}$.).

Nach dem Ausscheiden des älteren Fabrikinspektoren Wörrishoffer, mit dem Marie Baum gut zusammen gearbeitet hatte, wurden ihr durch dessen Nachfolger, einen Dr. Bittmann, dermaßen viele Steine in den Weg gelegt, daß sie ihre Arbeit für nicht mehr durchführbar hielt, sie geradezu in ihr Gegenteil verkehrt sah. Bittmann hatte erreicht, daß sie, die bis 1906 zunächst gleichberechtigt neben dem männlichen Fabrikinspektoren hatte arbeiten können, ihm untergeordnet wurde, wodurch jüngere Amtskollegen ihre Berichte »korrigieren « konnten. Sie mochte sich derartige männliche Gockeleien aus Prinzip nicht bieten lassen. Sie verlangte, daß sie den männlichen Fabrikinspektoren wieder gleichgestellt würde. Ohne Erfolg.

Max Weber schrieb ihr mehrfach und beschwor sie durchzuhalten: »Wo ist, wenn Sie fortgehen, jemand der so tapfer diesen Kampf mit subalterner >Männlichkeit ‘ aufnimmt. Nach 2, 3 Jahren liegen die Dinge anders; dann ist die Position so gefestigt, $\mathrm{da} B$ sie auch in schwächere Hände gelegt werden kann. ${ }^{4}$ Aber Marie Baum hielt nur noch kurze Zeit aus. Bei ihrer Entlassung 1907 ergriff Max Weber in Leserzuschriften in der "Frankfurter Zeitung " Partei für sie. ${ }^{5}$ Am 24. Januar 1907 brachte die Frankfurter Zeitung eine lange Einschätzung des Konflikts von Max Weber, in er zu dem Resultat kam, da $\beta$ das Experiment der weiblichen 
Fabrikinspektorin mit der Kündigung Marie Baums gescheitert sei und zwar gescheitert allein an - wie er sich ausdrückte - »männlichen Geschlechtseitelkeiten «, die zugelassen hatten, daß die Fabrikinspektorin dem männlichen Kollegen nur deshalb unterstellt worden war, weil dieser um seine Reputation unter seinen Geschlechtskollegen in der Behörde besorgt war. ${ }^{6}$

Marie Baum zog zu ihrer Freundin Else Jaffé-Richthofen nach Heidelberg. Beide gemeinsam widmeten ihre Zeit nun dem Ständigen Ausschuß zur Förderung der Arbeiterinnen-Interessen beim Bund Deutscher Frauenvereine. Als Else JafféRichthofen 1910 in einem Vortrag auf der »Zweiten Konferenz zur Förderung der Arbeiterinnen-Interessen «, die der Ständige Ausschuß ausgerichtet hatte, einen Überblick zur »Frau in der Gewerbe-Inspektion « gab, fiel ihr Resumé nicht nur negativ aus. Sie kritisierte, daß die Strukturen der Administration den Frauen wenig Chancen gaben, da $B$ - in ihren Worten - im »bürokratischen Organismus das Eigentliche der Frau erstarrt und nicht zur Geltung « kommen kann. Sie hoffte aber nach wie vor, daß akademisch geschulte Frauen mit unabhängiger Stellung doch immerhin einiges würden erreichen können.

Marie Baum schrieb nicht nur, sondern ging auch wieder in die praktische Sozialarbeit: 1907 übernahm sie - wiederum in einer Pionierinnenrolle - die erste städtische Säuglingsfürsorgestelle in Düsseldorf (1907-1916). 1917 wurden sie und Gertrud Bäumer zu Rektorinnen der neu gegründeten sozialen Frauenschule Hamburgs berufen. Später versuchte sie noch einmal, den Anliegen der Frauenbewegung in Ministerialdiensten zum Durchbruch zu verhelfen, gab aber wiederum wegen Behinderung ihrer Arbeit durch die Bürokratie auf. Von 1928 bis 1939 unterrichtete sie an der Universität Heidelberg als Dozentin für »Soziale Fragen«, bis sie wegen ihrer jüdischen Großmutter, einer Mendelssohn-Bartoldy, entlassen wurde.

Ab 1928 beteiligte sich Marie Baum an der von Alice Salomon herausgegebenen Reihe, Studien $\mathbf{z u}$ »Bestand und Erschütterung der Familie der Gegenwart«, detaillierten empirischen Studien zur Situation von Fabrikarbeiterinnen mit Familie, Jungarbeiterinnen usw., die bis heute lesenswert sind. Mit Alice Salomon verfaßte Marie Baum 1930 den Band »Das Familienleben der Gegenwart - 182 Monographien «, mit Alix Westerkamp 1931 »Rhythmus des Familienlebens - Das von einer Familie täglich zu leistende Arbeitsprogramm«. Diese Bände sind von der heutigen deutschen Universitäts-Soziologie noch kaum wieder entdeckt, vielleicht weil sie sich gegen die Einseitigkeiten der damaligen Universitätsempirie wandten. Von nordamerikanischen Historikern sind die Bände als ergiebige Quellengattung zur Geschichte der Familie in der Weimarer Zeit systematisch gewissermaßen ausgeweidet worden (William Hubbard 1983).

Erfahrungen mit der Gnadenlosigkeit von »Apparaten « machte Max Weber besonders während seiner Militärzeit und im Ersten Weltkrieg, aber wir dürfen annehmen, da $\beta$ auch die Erfahrungen der damaligen Frauenbewegung in die Theorien der Brüder Weber zur drohenden Verbürokratisierung der Welt nicht unerheblich waren. Die Diskussion der Behinderung ihrer sozialen Reformen durch die staatliche Bürokratie war in den Zeitschriften der Frauenbewegung Dauerthema. Bei Max Weber bildete sich die These von einem universellen Rationalisierungsproze $B$ erst nach und nach heraus (Martin Riesebrodt 1980) 
parallel zu den Debatten über sozial motivierte Staatseingriffe in den Wirtschaftsproze $B$.

Die empirischen Studien über "Auslese und Anpassung der Arbeiterschaft«, die Alfred und Max Weber im Rahmen des Vereins für Sozialpolitik ab 1906 anregten, sind zweifelsohne Erfolg des Heidelberger Gesprächskreises um Marianne Weber. ${ }^{7}$ Es war nicht einfach, Menschen zu finden, die bereit waren, die umfangreichen Befragungen durchzuführen. Wohl nicht zufällig wurden dafür vom Verein für Sozialpolitik, der 1901 erstmals eine weibliche Teilnehmerin zu seiner Jahrestagung zugelassen hatte, zur Hälfte Frauen beauftragt: Marie Bernays, Rosa Kempf, Elise Hermann und Elisabeth Hell, Autorinnen, die in den damaligen Frauenbewegungszeitschriften zahlreiche Diskussionsbeiträge lieferten, neben anderem zur Frage der Sozialarbeit. Die Untersuchungen wurden weithin bekannt. Vor allem die Untersuchungen von Marie Bernays über die Arbeiterinnen einer Spinnerei und Weberei in Gladbach und die von Rosa Kempf über junge Fabrikmädchen in München erfuhren viel Beachtung. Die Frauenorganisationen machten seit einigen Jahren auf die Misere etwa der Heimarbeiterinnen aufmerksam. Angesichts der staatlichen Bürokratie waren ihre Reformversuche aber immer wieder gefährdet, in ihr Gegenteil verdreht zu werden.

Angesichts der konservativen Wende in der Sozialpolitik Ende des ersten Jahrzehnts des 20. Jahrhunderts plädierte Max Weber für eine wertfreie Wissenschaft. Daraufhin entstand neben dem Verein für Sozialpolitik 1909 die Deutsche Gesellschaft für Soziologie (Dieter Lindenlaub 1967, $385 \mathrm{ff}$.). So verständlich diese Umkehr zur Wertfreiheit war, so fragt sich doch, ob sie nicht - wie auch in Chicago - auf Kosten des Kontakts zur sozialen Praxis ging, zumal man sich damit in gewisser Weise erneut in exklusiven Männerhochburgen einigelte, auch wenn einige Frauen nun zuhören durften. Allerdings flohen auch »Frauenrechtlerinnen « von der Politik in die Sozialforschung, wie wir schon von Marie Baum hörten. Alice Salomon richtete ihre Forschungsreihe "Bestand und Erschütterung der Familie der Gegenwart 1928 in dem Moment ein, als sie aufgab, gegen die seit der staatlichen Anerkennung ständig in ihre Hochschule hineinregierende Bürokratie anzukämpfen. Sie konzentrierte ihre Energien auf die neu ins Leben gerufene Weiterbildungsakademie für bereits »praktizierende«Sozialarbeiterinnen, die zugleich als Forschungsinstitution diente.

Zur Zeit der Jahrhundertwende war der intellektuelle Austausch zwischen den Wissenschaftlern und den Feministinnen deshalb so »befruchtend «, weil die Frauen als Aktivistinnen der Frauenbewegung und Praktikerinnen der neuen sozialen Arbeit vielfältige Erfahrungen »vor Ort« machten. Sie brachten infolge der am eigenen Leibe erfahrenen Praxis neue Fragestellungen ein, die ihnen als Wandernde zwischen den Welten gekommen waren. Sie entsprachen damit der Figur des Außenseiters, die Robert Park und Georg Simmel nicht nur als Opfer sondern auch als potenteste Zeitdiagnostiker ausgemacht hatten (Rolf Lindner 1990, $210 \mathrm{ff}$.).

Die Frauenrechtlerinnen hatten dem bürgerlichen Weiblichkeitsideal zu einem Gutteil den Rücken gekehrt. Sie scheuten sich nicht, mit Geächteten wie mit unehelichen Müttern oder Prostituierten den Kontakt aufzunehmen. Sie gingen für drei Monate in die Fabrik, um über die Situation von Arbeiter(inne)n berichten zu können. Sie wagten sich in extreme Männerhochburgen wie Universitäten und 
Stadtverwaltungen. Sie waren Ausnahmen und Randfiguren, die kaiserzeitliche Konventionen in Frage stellten. Sie waren maßgeblich daran beteiligt, ein neues Berufsfeld und eine neue wissenschaftliche Disziplin zu schaffen. Noch war die Soziologie kein eigenes Fach, die ersten Berufungen fanden in Deutschland erst 1917 nach Frankfurt am Main und Straßburg statt. Auf die Notwendigkeit empirischer Sozialforschung hatte neben der Arbeiterbewegung (`Soziale Frage ‘) die Frauenbewegung ( $>$ Soziale Arbeit $\iota$ ) aufmerksam gemacht. Durch die enge Verschwisterung von Sozialer Arbeit und Recherche entstand die Sozialforschung, etablierte sich die Soziologie.

Die Männer, mit denen die Frauen in der Gesellschaft für ethische Kultur, auf den Abenden der Frauen- und Mädchengruppen für soziale Hilfsarbeit, bei den Webers in Heidelberg zusammentrafen, waren ebenfalls Randständige: Juden, die in den preußischen Staatsdienst nicht aufgenommen wurden, Opfer der damaligen Sparpolitik (»Dozentenschwemme «), chancenlose Sozialdemokraten oder durch psychische Leiden von der Lehre abgehaltene Menschen wie Max Weber. So waren beide Gruppen geeignet, ihr starkes Interesse für soziale Reformen mit der nötigen Geduld für langwierige Auswertungen von Enquêten und soziale Erhebungen vor Ort zu verbinden.

Praktische Erfahrung und die Herausarbeitung theoretischer Positionen gingen dabei Hand in Hand. Wenn Max Weber für eine »verstehende Soziologie« plädierte, dann möglicherweise auch im Anschluß an seine Gespräche mit den Feministinnen seiner Zeit, die auf Grund ihrer praktischen Erfahrungen ein einfühlendes Verstehen für die durch "Entwurzelung «, Migration und durch desinteressierte Aufnahmegesellschaften Marginalisierten entwickelt hatten und konkrete Schritte zur Sozialreform einleiteten, die im »Kinderschutz «, in Jugendämtern, dem Reichswohlfahrtsgesetz sowie der Gründung von »Sozialen Frauenschulen « und der Einrichtung einer »Familienfürsorge« schließlich ihre »Materialisierung« erfuhren.

Bei der derzeitigen Großschreibung der empirischen Sozialforschung sollte dieser enge Zusammenhang von empirischer Sozialforschung zu Sozialarbeit und Sozialpädagogik nicht vergessen werden. Statt über "Langzeitstudent(inn)en « zu klagen, sollte geprüft werden, ob nicht viele der etwas länger Studierenden »nebenbei« ein Frauenhaus, einen Mädchenladen, eine Wohngemeinschaft für jugendliche Treber oder Deutschkurse für durch ihre Fluchtgeschichte traumatisierte Pakistaninnen oder Bosnierinnen ins Leben gerufen und ihre Erfahrungen eventuell sogar in einer kleinen Studie niedergeschrieben haben. Statt die Hochschulen für Sozialarbeit und Sozialpädagogik mittels ihrer Integration in Technikerhochschulen oder das Versetzen an Stadtränder zu marginalisieren, sollte vielmehr überlegt werden, ob die manchmal müde Universitätssoziologie sich nicht durch engeren Kontakt zu Sozialarbeit und Sozialpädagogik sinnvoll beleben lassen könnte. Wohl stand am Beginn der Soziologie die sozial motivierte Sozialreportage, aber die wäre ohne die Bereitschaft zu direktem sozialen Engagement seitens zahlreicher Feministinnen nicht denkbar gewesen. 


\section{Anmerkungen}

1 Mariannes Mutter war eine geborene Weber, eine Nichte von Max Webers Vater. Marianne Weber widmete Max Webers Mutter ihr erstes und wichtigstes Buch, "Ehefrau und Mutter in der Rechtsentwicklung«, Tübingen 1907.

2 Ich habe an anderer Stelle die dramatische Geschichte der ersten Prostituierten-Fürsorgerin, "Polizeiassistentin “ Henriette Arendt in Stungart, geschildert, deren Arbeit ebenfalls 1907 zum Erliegen kam, weil sie sich von der Polizeibehörde nicht in deren Sinne benutzen ließ. Elisabeth Meyer-Renschhausen 1983, siehe auch dies. 1986.

3 Vgl. etwa "Die Frauenbewegung « 2. Jg. 1896, 7.

4 Max Weber, Brief vom 23.8.1906, In: Gesamtausgabe, hrsg. von Rainer Lepsius und Wolfgang Mommsen, Tübingen: Mohr 1990, Bd. 5, Ab. II. Briefe 1906-1908, 147.

5 Ebd., Brief vom 23.1.1907, 218.

6 Max Weber in der Frankfurter Zeitung am 24.1. 1907, zit. nach dem Nachdruck dieses Briefes in "Die Frau«, 13. Jg. 1906/07, 373-375, 375.

$7 \mathrm{Zu}$ den ersten empirischen Untersuchungen von damaligen Frauenrechtlerinnen wie Elisabeth Gnauck-Kühne, Gertrud Dyrenfuth, Minna Wettstein-Adelt u.a. aus den späten 1890kr Jahren siehe Heinrich Herkner 1899, 238 f. Wenn Alfred Weber über das Sweating-System schrieb, dann nicht nur wegen der Weberschen Textilindustrie, sondern zweifelsohne auch infolge der Anregungen dazu durch Beatrice Webb geb. Potter und Florence Kelley. Vgl. dazu Dirk Käsler 1984. 349.

\section{Literatur (Auswahl)}

Archiv für Sozialwissenschaft und Sozialpolitik, Neue Folge des Archivs für Soziale Gesetzgebung und Statistik, begr. durch Heinrich Braun, hrsg. von Werner Sombart. Max Weber und Edgar Jaffé, 18. Bd. $1901=$ N.F. 1 . Bd. ff.

Baum, Marie (1906): Drei Klassen von Lohnarbeiterinnen in Industrie und Handel der Stadt Karlsruhe. Karlsruhe.

Baum, Marie (1927): Familienfurrsorge. Karlsruhe.

Baum, Marie (1950): Rückblick auf mein Leben. Heidelberg.

Baum, Marie und Alix Westerkamp (1931): Rhythmus des Familienlebens - Das von der Familie täglich zu leistende Arbeitspensum. Forschungen zu Bestand und Erschütterung der Familie in der Gegenwart. Berlin.

Bernays, Marie (1910): Auslese und Anpassung der Arbeiterschaft in der geschlossenen Großindustrie - dargestellt an den Verhälinissen der "Gladbacher Spinnerei und Weberei A.G." in Mönchen-Gladbach im Rheinland. Leipzig.

Bordin, Ruth (1981): Woman and Temperance - The Quest for Power and Liberty, 1873-1900. Philadelphia.

Buck, Gerhard (1982): Gemeinwesenarbeit und kommunale Sozialplanung. Berlin.

Conrad, Else [Else Kesten-Konrad] (1911): Artikel Verein für Sozialpolitik. In: Handwörterbuch der Staatswissenschaften. 3. gänzl. umgearbeitete Auflage, 8. Bd., Jena. 144-152.

Flexner, Eleanor (1978): Hundert Jahre Kampf - Die Geschichte der Frauenrechtsbewe gung in den Vereinigten Staaten. Frankfurt/M.

Gilcher-Holtey, Ingrid (1988): Max Weber und die Frauen. In: Max Weber-Ein Symposium. Hrsg. v. Christian Gneuss und Jürgen Kocka. München, 142-154.

Green, Martin (1976): Else und Frieda, die Richthofen-Schwestern. München.

Goldschmidt, Walter (1969/1972): Feldforschung. In: Wilhelm Bernsdorf (Hrsg.): Wörterbuch der Soziologie. Frankfurt/M., 3 Bde., Bd. 1, 226-228.

Jaffé-Richthofen, Else (1910): Die Frau in der Gewerbeinspektion. In: Schriften des ständigen Ausschusses zur Förderung der Arbeiterinnen-Interessen, Heft 3, Jena, 48-61

Jurczyk, Karin/Tatschmurat, Carmen (1985): Leben und Arbeiten der Industriearbeiterinnen - Ein Stück Forschungsgeschichte. In: Frauenforschung: Beiträge zum 22. Deutschen Soziologentag, Dortmund 1984. Hrsg. v. der Sektion Frauenforschung in der DGS, Frankfurt/M./New York, 43-52. 
Jütte, Robert (1991): "Disziplin zu predigen ist eine Sache, sich ihr zu unterwerfen eine andere» Prolegomena zu einer Sozialgeschichte der Armenfürsorge diesseits und jenseits des Fortschritts. In: Geschichte und Gesellschaft. 17. Jg., 92-101

Hermann, Elise (1912): Auslese und Anpassung der Arbeiterschaft in der geschlossenen Großindustrie - Dargestellt an den Verhälmissen einer Luckenwalder Wollhutfabrik Leipzig.

Herkner, Heinrich (1899): Das Frauenstudium der Nationalökonomie in Zürich. In: Archiv für Soziale Gesergebung und Statistik, 13. Bd., 227-254.

Hubbard. William H. (1983): Familiengeschichte - Materialien zur deutschen Familie seit dem Ende des 18. Jahrhunderts. München.

Kaplan, Marion (1981): Die Jüdische Frauenbewegung in Deutschland. Hamburg.

Käsler, Dirk (1984): Die frühe deutsche Soziologie 1909 bis 1934 und ihre Entstehungsmilieus. Opladen.

Kelley, Florence (1897): Die weibliche Fabrikinspektion in den Vereinigten Staaten. In: Archiv fur Soziale Geseizgebung und Statistik, 11. Jg., 128-142.

Kelley, Florence (1898): Sweating-System. In: Archiv für Soziale Gesetzgebung und Statistik, 12. Jg., 207-232.

Kelley, Florence (1899): Die Italiener Chicagos. In: Archiv furr Soziale Gesetzgebung und Statistik, 13. Jg., 291-313.

Kempf, Rosa (1911): Das Leben junger Fabrikmädchen in München Leipzig.

Lepenies, Wolf (1985): Die drei Kulturen - Soziologie zwischen Literatur und Wissenschaft. München/Wien.

Lexis, Wilhelm (1910): Artikel "Kathedersozialismus«. In: Handwörterbuch der Staatswissenschaften. 3. gänzl. umgearbeitete Auflage, 5. Bd., Jena, 804-806.

Lindenlaub, Dieter (1967): Richtungskämpfe im Vere in fïr Sozialpolitik. Wiesbaden, 2 Bde. (Beihefte der Vierteljahreshefte für Sozial- und Wirtschaf tsgeschichte).

Lindner, Rolf (1990): Die Entdeckung der Stadtkultur-Soziologie aus der Erfahrung der Reportage. Frankfurt/M.

MacKenzie, Jeanne (1979). A Victorian Counship - The Story of Beatrice Potter and Sidney Webb. London.

Meyer-Renschhausen, Elisabeth (1989): Weibliche Kultur und soziale Arbeit - Eine Geschichte der Frauenbewegung 1810-1927 am Beispiel Bremens. Köln/Wien.

Meyer-Renschhausen, Elisabeth (1986): Die weibliche Ehre - Ein Kapitel aus dem Kampf von Frauen gegen Polizei und Ärzte. In: Frauen-Körper-Medizin, hrsg. von Johanna Geyer-Kordesch, Annette Kuhn, Düsseldorf, 80-101.

Meyer-Renschhausen, Elisabeth (1983): Der Männerhaß der Polizeimatrone - Zur Sittlichkeitsbewegung. In: Courage, 8. Jg., 41-46.

Mommsen, Wolfgang J. (1981): Die antinomische Struktur des politischen Denkens Max Webers. In: Historische Zeitschrift 233, 35-64.

Mülder-Bach, Inka (1987): "Weibliche Kultur « und "stahlhartes Gehäuse «-Zur Thematisierung des Geschlechterverhältnisses in den Soziologien Georg Simmels und Max Webers. In: Sigrun Anselm, Barbara Beck (Hrsg.): Triumph und Scheitern in der Metropole - Zur Rolle der Weiblichkeit in der Geschichte Berlins. Berlin, 115-140.

Müller, Wolfgang C. (1982): Wie Helfen zum Beruf wurde - Eine Methodengeschichte der Sozialarbeit. Weinheim/Basel.

Peters, Dietlinde (1984): Mütterlichkeit im Kaiserreich. Bielefeld.

Riesebrodt, Martin (1980): Ideen, Interessen, Rationalisierung: Kritische Anmerkungen zu F. H. Tenbrucks Interpretation des Werkes Max Webers. In: Kölner Zeitschrifi für Soziologie und Sozialpsychologie, 32. Jg., 111-129.

Salomon, Alice (1983): Charakter ist Schicksal - Lebenserinnerungen. Hrsg. v. Rolf Landwehr und Rüdeger Baron, Weinheim/Basel.

Schwerin, Jeanette (1894/1895): Arbeiterschutz und Fabrikinspektion. In: Die Frau, 2. Jg., 263-268.

Simmel, Georg (1906): Zur Soziologie der Armut. In: Archiv für Soziale Gesetzgebung und Statistik, 22. Bd., $1-30$.

Simmel, Georg (1985): Schriften zur Philosophie und Soziologie der Geschlechter. Hrsg. von Heinz-Jürgen Dahme und Klaus Christian Köhnke. Frankfurt/M. 
Stoehr, Irene (1990): Emanzipation zum Staat? - Der Allgemeine Deutsche Frauenverein (Deutscher Staatsbürgerinnenverband) 1893 - 1933. Pfaffenweiler.

Webb, Beatrice (1988): Meine Lehrjahre. Frankfurt/M. (1926).

Weber, Marianne (1926): Max Weber - Ein Lebensbild. Tübingen

Weber, Marianne (1948): Lebenserinnerungen. Bremen.

Weber, Max (1922): Wirtschafi und Gesellschafi. Hrsg. v. Marianne Weber. Tübingen.

Weber, Max (1990): Gesamtausgabe. Hrsg. von Rainer Lepsius und Wolfgang Mommsen. Tübingen.

Wendt, Wolf Rainer ( $\left.{ }^{2} 1985\right)$ : Geschichte der sozialen Arbeit. Stuttgart.

Für anregende Gespräche und Interesse an diesem Aufsatz danke ich Gabriele Czarnowski, Helgard Kramer und Carol Hagemann-White. 